\title{
Effects of size at birth, gestational age and early growth in preterm infants on glucose and insulin concentrations at 9-12 years
}

\author{
M.S. Fewtrell ${ }^{1}$, C. Doherty ${ }^{2}$, T.J. Cole ${ }^{1}$, M.Stafford ${ }^{3}$, C.N.Hales ${ }^{1}$, A. Lucas $^{4}$ \\ ${ }^{1}$ MRC Human Nutrition Research, Cambridge, UK \\ ${ }^{2}$ University Department of Clinical Biochemistry, Addenbrooke's Hospital, Cambridge, UK \\ ${ }^{3}$ Department of Epidemiology and Public Health, UCL Medical School, London, UK \\ ${ }^{4}$ MRC Childhood Nutrition Research Centre, Institute of Child Health, London, UK
}

\section{Abstract}

Aims/hypothesis. To test the hypothesis that small size for gestation and poor postnatal growth in preterm infants is associated with higher fasting and post-load plasma glucose and insulin concentrations at 9-12 years of age.

Methods. Prospective follow-up at 9-12 years of 385 preterm children with birth weight less than $1850 \mathrm{~g}$, who had anthropometry recorded at birth, 18 months and 7 years. Fasting plasma glucose, insulin, proinsulin and 32,33 split proinsulin concentrations and glucose and insulin concentrations 30 min after a standard glucose load were measured.

Results. Post-load glucose concentrations were negatively related to birth weight, independently of gestation or subsequent growth. Fasting split proinsulin and 30-min insulin concentrations were highest in children who showed the greatest increase in weight centile between birth and current follow-up, regardless of gestation. When weight during childhood was included, birthweight centile was, however, no longer statistically significant: concentrations of fasting, split, proinsulin and 30-min insulin were highest in those children who had shown the greatest increase in weight centile between 18 months of age and current follow-up, with no evidence of a greater effect in those who were smallest at 18 months.

Conclusion/interpretation. Our findings suggest that fetal growth influences plasma glucose 30 min after a glucose load in preterm children at 9-12 years. In contrast, childhood weight gain is the most important factor influencing insulin concentrations and this effect is the same regardless of early size. [Diabetologia (2000) 43: 714-717]

Keywords Type II diabetes mellitus, preterm infant, childhood, glucose, insulin, birth weight, growth.
People with low birth weights could be at increased risk of developing impaired glucose tolerance (IGT) and Type II (non-insulin-dependent) diabetes mellitus later in life and this effect could be augmented in those who become overweight as adults $[1,2]$. Impaired glucose tolerance or fasting hyperinsulinaemia have been shown in children during the first decade of life [3-6] and in young adults who had low birth

Received: 25 October 1999 and in revised form: 22 February 2000

Corresponding author: Dr M. Fewtrell, MRC Childhood Nutrition Research Centre, Institute of Child Health, 30 Guilford Street, London WC1N 1EH, UK

Abbreviations: SDS, Standard deviation score. weight [7]. The influence of postnatal growth on subsequent glucose and insulin concentrations has received little attention, although two studies $[1,6]$ suggest that growth during infancy or childhood as well as during fetal life can influence the risk of later IGT and Type II diabetes.

Most reports involve subjects born at term. Preterm infants are, however, an interesting group in which to examine the effect of nutrition and growth independently of maternal influences during the last weeks of pregnancy; they often grow poorly because of poor nutritional intake but they are released from maternal or placental constraints on growth (nutritional, vascular or hormonal) during this period. They also show greater variation in postnatal growth than would be seen in children born at term. 
We measured fasting glucose and insulin and the response to an oral glucose load in preterm children at 9-12 years, to examine: the relation between gestation and size for gestation and later glucose and insulin concentrations and the effect of postnatal growth on later glucose and insulin concentrations.

\section{Subjects and methods}

Children were recruited from a cohort of 926 infants from five neonatal units in the United Kingdom [8] enrolled in a randomised multicentre trial of nutrition in preterm infants between 1982 and 1985. All weighed less than $1850 \mathrm{~g}$ at birth. Weight was recorded at 18 months and 7 years post-term.

Of these children 503 (61.9\% of survivors) participated in the current study at 9-12 years of age and were seen by one of two investigators at their local hospital or surgery. Blood was taken between 0900 hours and 1800 hours (median 1230 hours) after a minimum 6-h fast. Eutectic mixture of local anaesthetics (EMLA) (Astra Pharmaceuticals, Slough, UK) cream was applied before venepuncture. If the child consented, the needle was taped in place and a glucose load $(1.75 \mathrm{~g} /$ $\mathrm{kg}$ up to a maximum $75 \mathrm{~g}$ ) given as lucozade (Smith Kline Beecham, King's Langley, UK). A second blood sample was obtained after $30 \mathrm{~min}$. Samples were separated and frozen at $-70^{\circ} \mathrm{C}$ within $45 \mathrm{~min}$. Weight and height were measured. Pubertal status was self-assessed from photographs. Details of any relatives with Type II diabetes were recorded.

Ethical approval was obtained from the committees of the Dunn Nutrition Unit and the original participating centres. Written parental consent was obtained and verbal consent from each child.

Plasma glucose and insulin concentrations were measured as described previously [1].

Statistics. Glucose concentrations were normally distributed. Insulin concentrations were normalised by transformation to natural logarithms. Standard deviation scores (SDS) for birth weight, weight at 18 months, weight at 7 years and current weight and height were calculated using British growth reference data.

To examine the influence of size at birth and subsequent growth on later glucose and insulin, four sequential regression models were used in which biochemical measurements were regressed on: (1) current size, (2) early size, (3) early and late size and (4) early and late size plus the interaction between the two [9]. A $p$ value of less than 0.05 was regarded as significant.

\section{Results}

385 children provided a fasting blood sample [200 (51.8\% ) boys] and 291 (50.5\% boys) provided a sample 30 min after the glucose load. The mean birth weight of children providing blood was 1387 (SD 298, range 622-1847) g, birth weight SDS -0.90 (SD 1.25, range -4.35-1.94) and gestation 31.1 (SD 2.7, range 25-39) weeks.

Current size and biochemical measurements are shown in Table 1 . Of the subjects seen $46(23 \%)$ boys and $78(42 \%)$ girls were pubertal (Tanner Stage
2 or above). Girls had statistically significantly higher insulin concentrations than boys and boys had higher fasting and 30-min glucose. Sex differences persisted in all subsequent analyses; they were reduced but not removed when pubertal children were excluded. Age and time of sampling were independently associated with glucose and insulin and were included in all regression models.

Weight SDS showed a strong positive relation with fasting and 30-min insulin $(p<0.001)$ and with fasting glucose $(p=0.007)$. Relations between height SDS and biochemical measurements were similar. The 30min glucose was negatively associated with current weight and, more strongly, with current height.

Birth weight was negatively associated with 30min glucose (coefficient $-0.0012, p=0.009$ ) before and after adjusting for gestation. Birthweight SDS was negatively associated with 30-min glucose (coefficient $-0.28, p=0.013$ ) but only after adjusting for gestation, which was itself not significant. Fasting glucose showed no relation with birth weight SDS or birthweight, before or after adjusting for current size.

There was no significant relation between birthweight SDS and insulin concentrations. After adjusting for current size, both split proinsulin and 30-min insulin were, however, negatively associated with birthweight SDS [regression coefficients (95\% CI) $-7.1(-13.6$ to -0.7$) ;-7.9(-14.6$ to -1.1$)$, respectively adjusted for sex, gestation, sample time and current age]. Thus, for children with the same birth weight SDS, those with the greatest increase in weight SDS between birth and current follow-up have the highest insulin. There was no significant interaction between birth weight and current-weight SDS on insulin concentrations. Gestation and thinness at birth were not associated with later glucose or insulin and there was no interaction between birthweight SDS and gestation.

Current weight had a much greater effect on plasma insulin than birth weight. A 1 SD increase in current-weight SDS was associated with a $25.6 \%$ increase in 30-min insulin and a $31.7 \%$ increase in split proinsulin whereas the effect of a $1 \mathrm{SD}$ increase in birth weight SDS was to decrease 30-min insulin by $7.9 \%$ and split proinsulin by $7.1 \%$.

Growth during the immediate postnatal period (but still before term) was unrelated to later glucose or insulin, and adjusting for it did not alter the effect of birthweight SDS already described. Of the children studied at 9-12 years [366] had weight recorded at 18 months corrected age and 367 at 7 years. Birth weight SDS, 18-month and 7-year weight SDS were incorporated in a stepwise regression model with current size (Table 2). Fasting glucose and all insulin measurements showed a positive relation with current weight SDS. All insulin concentrations showed significant negative relations with 18 -month weight SDS and, for split and proinsulin, also with 7-year 
Table 1. Characteristics of study cohort

\begin{tabular}{|c|c|c|c|c|c|c|}
\hline & \multicolumn{2}{|c|}{ Boys and girls } & \multicolumn{2}{|c|}{ Boys } & \multicolumn{2}{|c|}{ Girls } \\
\hline & $n$ & mean (SD) & $n$ & mean (SD) & $n$ & mean (SD) \\
\hline Age (years) & 385 & $11.27(0.87)$ & 200 & $11.24(0.89)$ & 185 & $11.30(0.85)$ \\
\hline Height SD score & 385 & $-0.38(1.1)$ & 200 & $-0.32(1.1)$ & 185 & $-0.45(1.1)$ \\
\hline BMI SD score & 385 & $-0.15(1.2)$ & 200 & $-0.08(1.2)$ & 185 & $-0.23(1.2)$ \\
\hline $\begin{array}{l}\text { Fasting } \\
\text { glucose }(\mathrm{mmol} / \mathrm{l})\end{array}$ & 358 & $4.76(0.40)$ & 185 & $4.81(0.4)$ & 173 & $4.72(0.4)^{\mathrm{a}}$ \\
\hline Proinsulin $(\mathrm{pmol} / \mathrm{l})$ & 384 & $4.14(2.8,6.3)$ & 200 & $3.78(2.4,5.3)$ & 184 & $4.53(2.9,7.0)^{\mathrm{b}}$ \\
\hline 32, 33-split proinsulin ( $\mathrm{pmol} / \mathrm{l})$ & 385 & $6.36(3.9,10.0)$ & 200 & $5.37(3.2,8.0)$ & 185 & $7.59(4.4,13.0)^{\mathrm{b}}$ \\
\hline $\begin{array}{l}30 \mathrm{~min} \text { post-load } \\
\text { glucose }(\mathrm{mmol} / \mathrm{l})\end{array}$ & 279 & $9.03(1.74)$ & 142 & $9.37(1.9)$ & 137 & $8.67(1.5)^{\mathrm{b}}$ \\
\hline Insulin $(\mathrm{pmol} / \mathrm{l})$ & 291 & $365.0(254,534)$ & 147 & $333.62(239,441)$ & 144 & $399.85(284,590)^{\mathrm{a}}$ \\
\hline
\end{tabular}

${ }^{\mathrm{a}} p \leq 0.05,{ }^{\mathrm{b}} p \leq 0.005$. Values $=$ mean $(\mathrm{SD})$ for glucose and geometric mean $\left(25^{\text {th }}, 75^{\text {th }}\right.$ centiles $)$ for insulin concentrations

Table 2. Glucose and insulin concentrations and growth during childhood ${ }^{\mathrm{a}}$

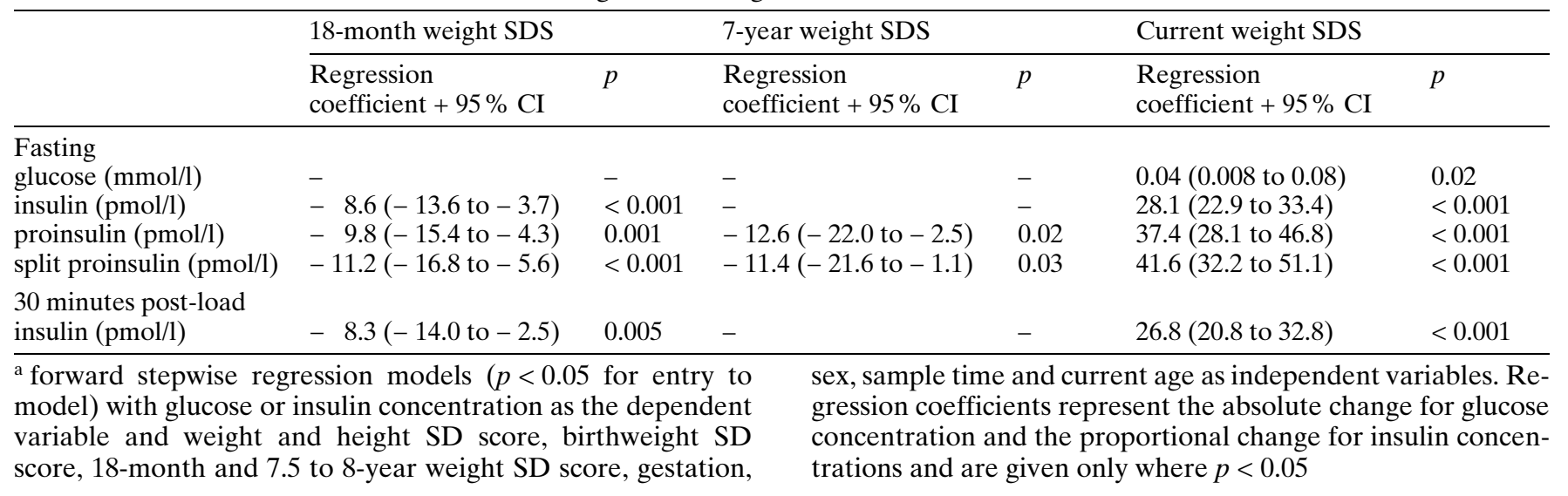

weight SDS. There was, however, no relation between birth weight SDS and insulin after adjusting for later growth. In this model 30-min glucose showed no relation with weight SDS at any age but was negatively related to birth weight. Thus, although current weight strongly and positively predicts insulin concentrations at 9-12 years, for children of equivalent current weight, insulin concentrations are higher in those with the greatest increase in weight SDS between 18 months and current follow-up. Of the 367 children $204(56 \%)$ had lower weight SDS at 18 months than they had at birth. The pattern of growth between birth and 18 months did not, however, influence insulin concentrations at 9-12 years.

There was a significant positive interaction between weight SDS at 18 months and current weight SDS on fasting and 30-min insulin and between weight SDS at 7 years and current weight SDS on split and proinsulin, suggesting that the effect of gain in weight SDS on insulin concentrations is greater in children who were heaviest earlier in childhood.

Results were not altered by excluding either firstly children whose mothers had gestational diabetes or insulin-dependent diabetes $(n=5)$ or secondly children born at more than 33 weeks gestation or by adjusting for social class at birth, parity, family history of Type II diabetes in a parent or grand-parent $(n=60)$ or current pubertal status (prepubertal versus pubertal).

\section{Discussion}

Low birth weight, whether due to prematurity or in utero growth restriction, was associated with higher plasma glucose $30 \mathrm{~min}$ after a glucose load in preterm children, independently of the pattern of growth during childhood. This finding, which is similar to that reported in term children [3-5], was independent of gestation suggesting that the critical period for this fetal programming effect could be before 25 weeks of gestation.

In contrast, plasma insulin concentrations were more strongly associated with the pattern of childhood weight gain than with growth in utero. Higher insulin concentrations were seen in children with the 
greatest increase in weight SDS (equivalent to upward centile crossing) between 18 months and current follow-up. That adjusting for size at an interim point in childhood removed the association between birth weight SDS and later insulin concentrations raises the possibility that, in other studies, birth weight might be acting as a proxy for a later, more influential weight (had the latter been available it might have altered the findings). Certainly our results shift the probable critical period for this particular effect forward from fetal life to childhood. A potential explanation for our findings is that the children who showed the greatest increase in weight centile between 18 months and the current follow-up were those who had entered puberty. Plasma insulin concentrations increase up to 13 years of age, with a peak just before the pubertal growth spurt in girls. Our results were, however, the same when pubertal children were excluded or after adjusting for pubertal status.

The interpretation of glucose and insulin concentrations in childhood cannot automatically be assumed to be the same as in adults. A full 2-h glucose tolerance test was considered unacceptable in these healthy children. In adults, glucose concentrations at $30 \mathrm{~min}$ are, however, related to those at $120 \mathrm{~min}$, and it is the immediate insulin response to a glucose load which determines glucose tolerance.

In conclusion, our results suggest that, at least in preterm children, there are different critical periods for the programming of glucose tolerance and insulin resistance. Glucose tolerance is associated with size at birth, whereas insulin concentrations are associated with the pattern of weight gain during childhood. Our findings could differ from those of other investigators for a number of reasons. Firstly, we studied preterm children whose in utero experience could have differed from that of term infants and who showed greater variation in postnatal growth. Secondly, we interpreted our data differently from previous investigators, including testing for interactions between growth measurements at different timepoints on later outcome as proposed recently [9]. Thirdly, we incorporated interim measures of growth during childhood which have either been unavailable or not analysed in many other studies. The finding that an increase in weight SDS during early to mid- childhood could be associated with hyperinsulinaemia is worrying in the context of an increasing prevalence of childhood obesity. Further investigation of other cohorts of children whose growth has been measured prospectively, including those born at term, is required to confirm or refute our findings.

Acknowledgements. We thank the children who participated in the study and their parents, the staff of the many hospitals and health centres for providing accommodation for the study, K. Taylor and I. Halsall for performing the biochemical assays and Dr N. Wareham for his comments on an earlier draft of the paper. The 9 to 12-year follow-up of this cohort was funded by the Medical Research Council (UK). M. Fewtrell and C. Doherty were supported by Medical Research Council (UK) Training Fellowships.

\section{References}

1. Hales CN, Barker DJ, Clarke PM et al. (1991) Fetal and infant growth and impaired glucose tolerance at age 64 years. BMJ 303: 1019-1022

2. Lithell HO, McKeigue PM, Berglund L, Mohsen R, Lithell UB, Leon DA (1996) Relation of size at birth to non-insulin dependent diabetes and insulin concentrations in men aged 50-60 years. BMJ 312: 406-410

3. Law CM, Gordon GS, Sheill AW, Barker DJ, Hales CN (1995) Thinness at birth and glucose tolerance in sevenyear-old children. Diab Med 12: 24-29

4. Yajnik CS, Fall CH, Vaidya U et al. (1995) Fetal growth and glucose and insulin metabolism in four-year-old Indian children. Diabet Med 12: 330-336

5. Whincup PH, Cook DG, Adshead F et al. (1997) Childhood size is more stingily related than size at birth to glucose and insulin levels in 10-11-year-old children. Diabetologia 40: 319-326

6. Crowther NJ, Cameron N, Trusler J, Gray IP (1998) Association between poor glucose tolerance and rapid post natal weight gain in seven-year-old children. Diabetologia 41: $1163-1167$

7. Robinson S, Walton RJ, Clarke PM, Barker DJ, Hales CN, Osmond C (1992) The relation of fetal growth to plasma glucose in young men. Diabetologia 35: 444-446

8. Lucas A, Gore SM, Cole TJ et al. (1984) A multicentre trial on feeding low birth weight infants: effects of early diet on early growth. Arch Dis Child 59: 722-730

9. Lucas A, Fewtrell MS, Cole TJ (1999) Fetal origins of adult disease - the hypothesis revisited. BMJ 319: 245-249

10. Hales CN, Barker DJP (1992) Type II (non-insulin dependent) diabetes mellitus: the thrifty phenotype hypothesis. Diabetologia 35: 595-601 